\section{$\underset{\text { hommes }}{\text { \& migrations }}$}

\section{Hommes \& migrations}

Revue française de référence sur les dynamiques

migratoires

$1296 \mid 2012$

Le Mexique dans les migrations internationales

\title{
La dynamique migratoire au Mexique
}

Un futur incertain

\section{Jorge Durand}

Traducteur : Bernardo Toro

\section{(2) OpenEdition \\ Journals}

\section{Édition électronique}

URL : http://journals.openedition.org/hommesmigrations/1509

DOI : 10.4000/hommesmigrations. 1509

ISSN : 2262-3353

\section{Éditeur}

Musée national de l'histoire de l'immigration

\section{Édition imprimée}

Date de publication : 1 mars 2012

Pagination : 12-21

ISSN : 1142-852X

Référence électronique

Jorge Durand, «La dynamique migratoire au Mexique », Hommes \& migrations [En ligne], 1296 | 2012, mis en ligne le 31 décembre 2014, consulté le 19 avril 2019. URL : http://journals.openedition.org/ hommesmigrations/1509; DOI : 10.4000/hommesmigrations.1509 


\section{La dynamique migratoire au Mexique Un futur incertain}

Par Jorge Durand, géographe et anthropologue, université de Guadalajara - Cide

L'émigration mexicaine en direction des États-Unis pourrait bien être arrivée à saturation. Le Mexique en pleine explosion démographique constituait pourtant une réserve de main-d'œuvre pour son puissant voisin du Nord. Désormais, la circulation migratoire entre le Mexique et les États-Unis se heurte à une frontière de moins en moins perméable. Depuis plusieurs années, la perception négative des migrants mexicains sur le territoire américain se conjugue à une politique dissuasive censée répondre à la crise économique. 
La tradition migratoire du Mexique vers les États-Unis a créé depuis un siècle un réseau très complexe de relations sociales, culturelles et familiales. À cette trame s'ajoutent les causes économiques et politiques qui sont à l'origine des flux migratoires. Cette tradition est liée à une politique très déterminée de la part des États-Unis visant à faire des Mexicains la principale réserve de main-d'ceuvre, à la fois disponible et jetable.

Mais les flux migratoires ne sont pas éternels. En Europe, le cas des pays comme l'Espagne, l'Italie et l'Irlande, entre autres, le prouvent. Même dans le cas du Mexique, l'un des pays avec le plus fort taux d'émigration au monde, on peut commencer à envisager la fin du phénomène. Deux conditions sont nécessaires pour que le flux migratoire s'arrête ou diminue : une baisse sensible de la natalité et une croissance économique soutenue. La première condition est déjà remplie au Mexique, la deuxième n'est remplie qu'à moitié puisque le rythme de la croissance économique est resté modéré au cours des deux dernières décennies et a même connu plusieurs chutes significatives. Au cours de cette période, l'émigration mexicaine aux ÉtatsUnis est tout de même arrivée à son plus haut niveau, même si elle décroît depuis trois ans. Cette baisse imprévue mérite une explication et une analyse approfondies. Tout d'abord, il faudrait déterminer s'il s'agit d'un processus normal propre aux flux migratoires qui arrivent à saturation et commencent naturellement à décroître (phénomène qu'on a appelé "la courbe migratoire"), ou bien d'une situation conjoncturelle dont la principale raison est la crise économique actuelle. Si tel était le cas, on peut estimer que le flux migratoire redeviendra stable dans les prochaines années.

\section{La dynamique migratoire}

L'émigration de Mexicains sans papiers aux États-Unis est arrivée à son plus haut niveau en 2007, quand le Pew Hispanic Center, qui mesure annuellement les flux migratoires Mexique-États-Unis, a évalué à 7 millions le nombre de migrants irréguliers. Par la suite, en se servant des mêmes indicateurs, on compte 6,8 millions de migrants en 2008, 6,7 en 2009 et 6,5 en 2010.

En réalité, on assiste à la convergence de plusieurs processus. Notons pour commencer que le flux de sortie s'est considérablement réduit. En 2005, on estimait à 500000 le nombre de migrants mexicains irréguliers qui chaque année traversaient la frontière pour se rendre aux États-Unis, alors qu'aujourd'hui, ils ne seraient plus que $150000^{(1)}$. En deuxième lieu, notons l'accroissement des renvois à la frontière et à l'intérieur du pays, situation qui a réduit le nombre total de migrants sans papiers. 
Le changement a été progressif mais significatif, la priorité étant désormais au contrôle de la migration, tant à l'intérieur du territoire qu'aux frontières. Les chiffres parlent d'eux-mêmes : en 1990, 30000 migrants ont été déportés depuis l'intérieur du territoire, un million à la frontière. Ces chiffres ont atteint respectivement 188000 et 1,5 million en 2000,393000 et 580000 en 2009 .

Enfin, la dernière cause de cette diminution de l'émigration irrégulière au cours des dernières années a été l'instauration de plusieurs types de visas pour les Mexicains. La politique qui consistait à refuser les visas pour des raisons économiques, refus qui rendait quasiment impossible son obtention, a été revue. En 1999, l'indice annuel d'accès aux visas était de 0,024, tandis que dix ans plus tard, en pleine crise économique, il était de $0,173^{(2)}$. Loctroi des visas temporaires $\mathrm{H} 2 \mathrm{~A}$ et $\mathrm{H} 2 \mathrm{~B}$ pour l'agriculture et les services, qui sont dans leur large majorité octroyés à des Mexicains, a particulièrement augmenté. En 2000, on a délivré 84754 visas, en 2008 le triple et en 2009 plus du double, $206144^{(3)}$.

Ces processus qui, en termes pratiques, consistent à ouvrir ou fermer les frontières, s'articulent avec d'autres facteurs et dynamiques, lesquels entraient en jeu de manière simultanée : les dynamiques démographique et économique qui sont structurelles et les dynamiques migratoires, professionnelles ou politiques, qui sont complémentaires.

\section{Les dynamiques démographique et économique}

La variable démographique est un élément clef dans ce processus. La population du Mexique a augmenté de manière géométrique, comme dirait Malthus, au cours d'un quart de siècle. Dans la période qui va de 1950 à 1975, la population est passée de 27,7 à 60,7 millions $^{(4)}$. Cette explosion démographique, qui a encore aujourd'hui des répercussions dans la structure de la population, a été qualifiée de "bond démographique". Dans ce contexte de croissance démographique explosive, les divers types de migration mexicaine ont constitué une issue de secours. De manière paradoxale ou concomitante, cette période a coïncidé avec une étape florissante pour l'économie, connue sous le nom de "miracle mexicain". Le Mexique a alors approfondi sa réforme agraire et impulsé la "révolution verte ${ }^{(5)}$.'À cette époque, le gouvernement avait réussi à articuler avec succès le modèle économique de substitution des importations ${ }^{(6)}$ avec un appareil d'État fort, sans alternance politique, qui avait le contrôle de l'économie et détenait les principales industries : électrique, pétrolière, communications, entre autres ${ }^{(\gamma)}$.

La croissance économique, génératrice d'emplois dans les villes, et l'immigration, aussi bien interne qu'internationale, ont permis d'équilibrer, dans une certaine 
mesure, les conséquences de la croissance explosive de la population, qui est arrivée à son plus haut niveau en 1965 avec un taux de fécondité de 6,9 enfants par femme. Pour établir un point de comparaison, notons qu'aux États-Unis, en plein baby-boom (1955), le taux global de fécondité a atteint 3,7 enfants par femme, alors que cette même année le Mexique atteignait $6,8^{(8)}$.

La croissance économique a compensé en partie les conséquences négatives de cette explosion démographique, mais elle a provoqué une redistribution de la population, de manière parfois chaotique, vers cinq pôles d'attraction. Tout d'abord, la ville de Mexico

Dans ce contexte
de croissance
démographique explosive,
les divers types
de migration mexicaine
ont constitué une issue
de secours.

s'est transformée en une mégapole. Dans les années quatre-vingt, on disait qu'elle était la ville la plus peuplée du monde, elle opérait comme un aimant très puissant pour la migration rurale-urbaine des États limitrophes du centre et du sud du pays, avec une importante population d'origine indienne $^{(9)}$. En deuxième lieu, la ville de Guadalajara, qui opérait comme un pôle d'attraction régional, commercial et de services pour les États voisins situés à l'ouest. Cette ville se vantait d'avoir atteint le million d'habitants à la fin des années soixante. Le rythme de la croissance urbaine a d'ailleurs été si fort qu'au cours de cette décennie la population a doublé. Personne ne semblait pour autant avoir une idée très claire des problèmes que cela pourrait entraîner dans le futur ${ }^{(10)}$. Un troisième centre d'attraction a été la ville de Monterrey qui grandit et s'industrialise rapidement et attire la population de la région nord-est ${ }^{(11)}$. En quatrième lieu, au milieu des années soixante, et comme résultat de la fin du programme Bracero, les villes frontalières, en particulier Tijuana, Ciudad Juarez et Nuevo Laredo, se sont mises à grandir. Elles ont reçu des immigrants de tout le pays qui, de manière très chaotique, voulaient satisfaire la demande de main-d'œuvre générée par le nouveau modèle économique ${ }^{(12)}$.

Enfin, à toute cette recomposition de la population mexicaine provoquée par des facteurs internes, il faut ajouter la dynamique externe engendrée par les États-Unis, lesquels demandent de manière urgente des travailleurs ruraux du Mexique pour l'économie de guerre, puis pour le développement agricole et finalement pour l'industrie et les services. En effet, le programme Bracero (1942-1964) a capté l'excédent de population rurale de la région occidentale du Mexique qui était particulièrement peuplée : Jalisco, Michoacan, Guanajuato, Zacatecas, San Luis Potosi et Durango, une région que l'on connaît comme celle de l'immigration historique ${ }^{(13)}$. Cet élan migratoire, généré par une incessante demande de main-d'œuvre de la part des ÉtatsUnis, s'est poursuivi jusqu'à aujourd'hui. 


\section{Cinq défis à relever}

En définitive, depuis la fin des années soixante-dix jusqu'à aujourd'hui, le pays a dû faire face à cinq graves problèmes : les crises économiques récurrentes, l'explosion démographique, la migration interne, l'immigration régulière et l'immigration internationale irrégulière.

Pour ce qui est du premier défi, il faut bien admettre que le pays n'a pas pu retrouver la voie de la croissance qu'il a connue au cours des années cinquante et soixante. À partir de la crise consécutive à la dévaluation de 1976, le même schéma s'est répété pratiquement tous les six ans, pour des raisons à chaque fois différentes. L'économie mexicaine s'est développée à un rythme de $2,3 \%$ en moyenne au cours des deux dernières décennies. Elle présente, par ailleurs, des altérations très marquées dues à l'endettement externe, à des facteurs internes, comme ladite "erreur de décembre" de 1994 (date de la dernière grande crise économique qui a conduit à une dévaluation au Mexique) et à une forte dépendance à l'égard de l'économie américaine, étant donné que la majorité des exportations sont tournées vers les États-Unis. Tant que la croissance économique n'est pas au rendez-vous, les facteurs d'expulsion continueront de déterminer les flux migratoires.

Le deuxième défi consiste à contrôler la croissance démesurée de la population. On peut affirmer que le Mexique est entré dans un processus de transition démographique et qu'il lui manque très peu pour descendre au niveau du seuil de renouvellement des générations. On remarque toutefois que les mesures correctrices ont toujours été prises quand le problème était déjà terminé. En 1974, on a promulgué la Loi générale de population, qui prétendait contrôler la croissance de la population, ainsi que l'émigration et l'immigration, en réalisant des "programmes de planification familiale à travers des services éducatifs et de santé publique" (art.2), en diminuant la mortalité(art. 3), en assujettissant l'immigration des étrangers aux modalités pertinentes (art.7) et, enfin, en restreignant l'émigration des citoyens quand l'intérêt national l'exige (art. 8).

Promulguée il y a trente-cinq ans, la Loi générale de population a été un succès. La planification familiale mise en place par la loi a permis de réduire le taux total de natalité, lequel est passé de 6,5 enfants par femme en 1975,à 2,2 en 2010. La mortalité infantile a aussi été réduite, passant de 69 sur mille en 1975 à 21 sur mille en $2010^{(14)}$. Les politiques publiques concernant la population ont été efficaces dans le contrôle de la natalité-mortalité et très peu performantes en matière d'immigrationémigration. C'est d'ailleurs une caractéristique commune à tous les pays démocratiques. Seules les dictatures ont réussi à contrôler par des moyens répressifs la migration, dans ses deux versants. En effet, alors que tous les efforts étaient consacrés à résoudre le problème de la croissance démographique, les questions de migration 
interne étaient laissées de côté. L'espace urbain et l'octroi de services (eau, lumière, drainage, pavage) ont été gérés de manière clientéliste et seulement dans la mesure où le temps, le budget et la patience du peuple le permettaient ${ }^{(15)}$. C'est la crise économique qui a freiné, dans une certaine mesure, l'exode rural vers les villes.

En quatrième lieu, et en accord avec la Loi générale de population, le Mexique a fermé les portes à l'immigration. Il l'a "maîtrisée" en prenant les mesures qu'il a estimées nécessaires et en rendant très difficiles l'immigration et la naturalisation des rares étrangers qui voulaient rester et s'intégrer. Ouvrir les portes du pays au moment où le principal problème était l'explosion démographique n'avait, en effet, aucun sens. D'après Pablo Yankelevich, la politique migratoire du Mexique postrévolutionnaire faisait la différence entre migrants "voulus et indésirables ${ }^{(16) "}$ "et se servait de l'article 33 de la Constitution pour exercer un contrôle strict sur la population étrangère.

Même si le pays se veut ouvert à l'immigration et généreux quant à l'octroi d'asile, la réalité de cette politique de portes fermées peut se vérifier à partir des données du recensement de 2010. On compte alors 961121 étrangers sur le sol national, ce qui représente $0,86 \%$ de la population globale. Une partie de ces étrangers, difficile à déterminer statistiquement, est composée d'enfants de Mexicains qui sont nés aux États-Unis et qui figurent dans ledit recensement comme des citoyens américains.

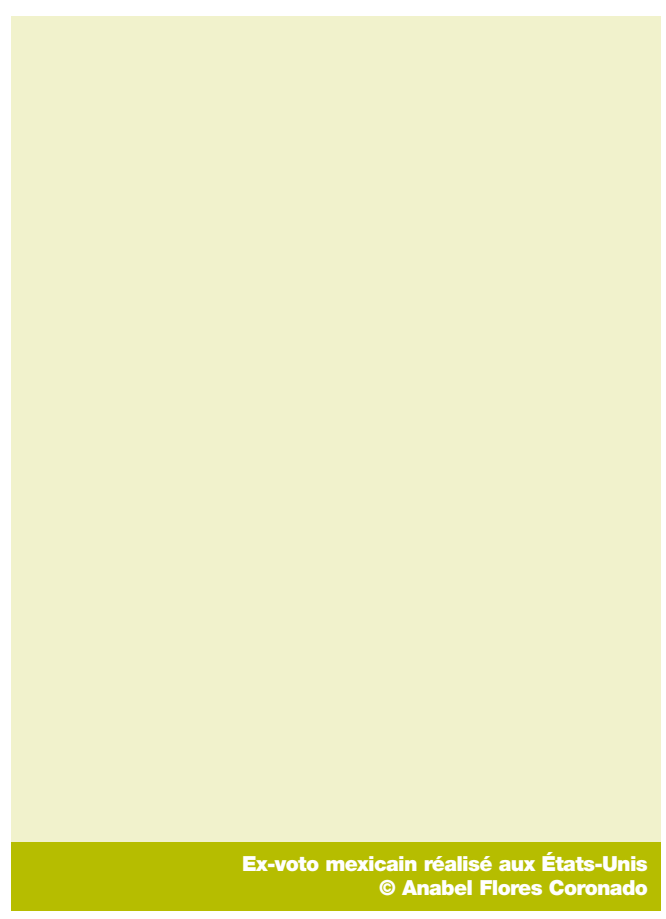
La proportion entre émigration et immigration est de 10 à 1 . En cinquième lieu, il faut remarquer que, si en matière d'immigration on avait mis en place une politique de contrôle, en matière d'émigration on appliquait le principe général qui stipulait qu'il fallait intervenir "quand l'intérêt national l'exige(ait)". Cette directive, qui faisait référence à un éventuel manque de maind'ceuvre qui ne s'est jamais vérifié, pouvait être comprise de plusieurs manières. Malgré ces cinq dispositifs, la pression démographique a continué d'augmenter, surtout quand la crise économique a touché le milieu rural et que la réforme agraire 
a cessé d'être une solution pour les nouvelles générations de paysans ${ }^{(17)}$. De cette manière, les flux migratoires vers l'intérieur du pays ont atteint leur limite et ont changé de sens en se dirigeant désormais vers l'extérieur du pays. Même les centres urbains sont devenus le lieu d'origine des futurs migrants. De cette manière, la migration internationale, qui était un phénomène principalement régional affectant la partie occidentale du Mexique, a pris une dimension nationale avec la participation de la totalité des États et de l'immense majorité des communes ${ }^{(18)}$.

La sortie des migrants irréguliers vers le Nord augmente année après année ; ceux-ci sont aussitôt absorbés par un marché du travail vorace et insatiable. Le Mexique n'a aucune alternative à proposer à l'émigration internationale, sauf celle qui consiste à recommencer ou renouveler le programme Bracero. C'est pour cette raison qu'on a appelé les politiques publiques mises en place à cette époque "des politiques de la non-politique". Elles consistaient, en effet, à laisser faire et à attendre que les États-Unis décident de la voie à suivre ${ }^{(19)}$.

L'apparition de nouveaux contingents d'émigrants internationaux provenant du centre et du sud du Mexique, qui ne trouvaient ni logement ni travail dans la ville de Mexico, a considérablement grossi le flux migratoire vers l'extérieur du pays. Cette dynamique a coïncidé avec un changement radical dans la politique d'immigration aux États-Unis. Le marché du travail américain et l'absence d'une véritable politique migratoire ont été un moteur puissant pour l'accroissement de ce flux qui se nourrissait des excédents de population du pays.

\section{Les réformes migratoires}

Pendant plus d'un siècle (1884-1986), les États-Unis avaient considéré les immigrants mexicains comme des travailleurs et non comme des immigrants. La viabilité du modèle poussait le gouvernement américain à les présenter comme des "travailleurs migrants sans papiers". Mais, en 1986, une loi (Immigration Reform and Control Act, IRCA) a régularisé la situation de 3,2 millions de migrants sans papiers, dont 2,3 millions étaient mexicains, en leur donnant un permis de séjour et ultérieurement une possibilité de naturalisation. Cette mesure a transformé totalement le modèle migratoire précédent, basé sur la circulation et le refus de papiers, pour inaugurer une nouvelle étape de régularisation à caractère définitif. En dehors de l'amnistie, la loi comprenait deux mesures complémentaires: le contrôle accru aux frontières et des sanctions appliquées aux employeurs.

Les conséquences de cette politique, beaucoup d'entre elles imprévues, ont été la fin de la circulation migratoire, la sédentarisation des migrants, la réunification familiale 
par des voies illégales ou informelles, l'allongement de la durée du séjour et le nonretour des migrants irréguliers. À mesure que le contrôle aux frontières se durcissait, les coûts et les risques de la migration clandestine augmentaient, ce qui a eu comme conséquence l'allongement de la durée du séjour, le non-retour et une augmentation importante du volume de la migration irrégulière installée aux États-Unis ${ }^{(20)}$. Les travailleurs migrants sont devenus des immigrés, des résidents migrants sans papiers. Une fois la frontière traversée, on pouvait travailler et vivre plus ou moins tranquillement à l'intérieur des États-Unis, du moins jusqu'au 11 septembre 2001. À partir de cette date une campagne officielle anti-immigrants a vu le jour, associant le thème de l'immigration à celui de la sécurité nationale, criminalisant ainsi l'immigrant et refusant toute forme de régularisation aux 11 millions de migrants irréguliers, dont 7 sont d'origine mexicaine.

Depuis 1993, la frontière entre le Mexique et les États-Unis a subi un changement radical. Auparavant ouverte et poreuse, elle est surveillée et même militarisée depuis 2001. Aujourd'hui, elle est devenue une barrière quasiment infranchissable.

Parallèlement au durcissement du contrôle des frontières, on a assisté à la persécution politique des migrants, lesquels ont commencé à être pris pour cible par beaucoup d'hommes politiques de droite. Depuis 1994, avec la proposition 187 de l'État de Californie, qui proposait d'affecter les migrants sans papiers dans les emplois de service, on a assisté à une véritable avalanche de lois et réglementations qui sanctionnent les migrants, telles que la loi de l'Arizona (SB 1070) ou celle de Alabama en 2011 (HB 56). Ces lois ont fini par créer un véritable climat de terreur et de persécution autour des migrants et de leurs familles.

\section{Conclusion}

La politique migratoire américaine, à la frontière et à l'intérieur des États-Unis, a commencé à porter ses fruits. Il a fallu attendre vingt ans, de 1987 à 2007, avant que le flux migratoire irrégulier ne commence à baisser. La politique dissuasive a opéré de manière constante au cours de la dernière décennie. Son but était de rendre la traversée de la frontière plus coûteuse et plus risquée. Aujourd'hui, son coût équivaut à mille jours de salaire minimum mexicain, ce qui a limité de manière considérable les intentions d'émigrer.

D'autre part, la crise de 2008 a coupé les voies de financement de l'entrée illégale sur le territoire américain, alimentées par les migrants déjà établis aux États-Unis. Ce financement, que nous avons appelé "systémique", a cessé d'être viable depuis la crise, à cause de la réduction des heures de travail, de la baisse des salaires et de la menace 
permanente du chômage. Dans ces conditions les migrants ne peuvent plus aider leurs parents à financer une aventure qui, en plus d'être coûteuse, peut s'avérer fatale. En effet, aux coûts, il faut ajouter les risques que suppose la traversée de la frontière par le désert. Au début des années quatre-vingt-dix, 75 \% des migrants traversaient la frontière par le couloir Tijuana-San Diego, mais le contrôle des frontières a fait dévier les points de traversée vers Sonora et Altar, où l'on enregistre un nombre important de morts chaque jour. Le risque de perdre la vie a dissuadé les migrants et leurs familles. À la rigueur du climat, il faut ajouter la situation de violence dans les régions frontalières depuis le jour où les mafias et le crime organisé ont commencé à racketter, à faire du chantage et à assassiner des migrants aussi bien mexicains que d'autres nationalités. Mais le risque va bien au-delà. Aujourd'hui, l'investissement fait pour entrer aux États-Unis n'est plus sûr, comme c'était le cas autrefois où, une fois la frontière traversée, le migrant sans papiers était presque sûr de trouver un emploi sans qu'on le dérange ou qu'on le renvoie chez lui.La persécution à l'intérieur du pays et le climat anti-immigrant qui règne aux États-Unis ont dissuadé les familles modestes d'investir des sommes importantes. De plus, l'immigrant capturé par la police est condamné à passer plusieurs mois en prison. La relation dépense-bénéfice a changé de manière substantielle.

D'un autre côté, des facteurs structurels aussi entrent en ligne de compte. Les familles avec deux enfants en moyenne ne se voient plus dans le besoin impérieux d'envoyer l'un de leurs enfants aux États-Unis pour diversifier et augmenter leurs revenus, ainsi qu'il arrivait aux familles nombreuses autrefois. Actuellement les jeunes vivent dans des familles bien plus réduites, les revenus locaux permettent tant bien que mal à leurs parents de subvenir à leurs besoins. La migration mexicaine n'a jamais été issue des secteurs les plus pauvres de la population. C'est encore moins le cas aujourd'hui. Malgré les graves problèmes économiques du Mexique, le très haut niveau d'inégalité, le sous-emploi et le chômage rampant, le produit intérieur brut par personne en 2010 était de 13900 dollars. Cette somme est semblable à celle que pourrait gagner un migrant qui travaille à temps complet, avec un salaire minimal de 7,5 dollars l'heure aux États-Unis. La comparaison est grossière, car le salaire minimal au Mexique est de 5 dollars par jour, mais dans la pratique le salaire réel d'un manceuvre ou d'une femme de ménage tourne autour de 20 dollars par jour. Pour beaucoup de Mexicains qui bénéficient d'un emploi, la différence de salaire entre les États-Unis et le Mexique s'est réduite au cours de la dernière décennie. En partie à cause de la nette dégradation du pouvoir d'achat aux États-Unis.

Un autre élément réside dans le changement radical de la perception du public nordaméricain face à la migration mexicaine. Depuis le début du XX $\mathrm{XX}^{\mathrm{c}}$ siècle et jusque dans les années quatre-vingt-dix, les Mexicains étaient concentrés dans les trois États de la Californie, du Texas et de l'Illinois, et ils se sont depuis dispersés dans tout le pays. Pour 
beaucoup d'Américains des nouvelles régions de destination de la migration, les Mexicains constituent un nouveau facteur qu'ils n'avaient pas pris en considération auparavant et qui vient rompre la traditionnelle relation entre Blancs et Noirs. Tous ces facteurs entrent en ligne de compte et notamment l'actuelle crise économique aux États-Unis. Mais la variable principale qui a déterminé et continuera à déterminer, à court comme à long terme, la réduction du flux migratoire irrégulier est le caractère répressif de la politique d'immigration américaine. La frontière a quasiment été scellée : aux rares immigrants qui parviennent à la traverser s'opposent ceux qui sont chaque jour renvoyés chez eux. Néanmoins, il est possible qu'une fois la porte fermée, celle-ci doive un jour être rouverte pour accueillir, de manière ordonnée et légale, la maind'cuvre dont a besoin l'économie américaine. Ce processus a déjà commencé avec l'importante augmentation de visas octroyés aux Mexicains en 2010.

\section{Traduit de l'espagnol par Bernardo Toro}

\section{Notes}

1. Jeffrey S. Passel, "Unauthorized immigrant population: national and state trends, 2010", Washington D. C.,

Pew Hispanic Center, February 1, 2011.

2. Mexican Migration Project (MMP), http://mmp.opr.princeton.edu/. 2011

3. US Department of Homeland Security (DHLS), Yearbook of Immigration Statistics, Estados Unidos, 2009.

4. ONU, World Population Prospects, New York, United Nations, 2007.

5. Cynthia Hewitt De Alcantara, La Modernización de la Agricultura Mexicana 1940-1970, México,

Siglo XXI Éditeurs, 1976.

6. Francisco Alba et al., "Industrialización sustitutiva y migración internacional: el caso de México", in Indocumentados, Mitos y Realidades, México, Le Colegio de México, 1979.

7. Patricia Arias (dir.), Industria y estado en la vida de México, Zamora, Le Colegio de Michoacán, 1990.

8. ONU, World Population Prospects, op. cit.

9. Claude Bataillon, Las regiones geográficas en México, México, Siglo XXI Éditeurs, 1986.

Claude Bataillon et Louis Panabiére, Mexico aujourd'hui. La plus grande ville du monde, Paris, Publisud, 1988.

10. Hélène Rivière D’Arc, Guadalajara y su Región, México, SepSetentas, 1973 ; Patricia Arias (dir.), Guadalajara la gran ciudad de la pequeña industria, Zamora, Le Colegio de Michoacán, 1985.

11. Jorge Balan, Harley L. Browinng et Elizabeth Jelin, Migración, Estructura Ocupacional y Movilidad Social. El Caso Monterrey, Unam, México, IIS, 1973.

12. Roland Travis, Industrie et politique à la frontière Mexique-USA Le Cas de Nuevo Laredo, 1966-1984, Paris, Éditions du CNRS, 1985.

13. Jorge Durand et Douglas S. Massey, Clandestinos. Migración México Estados Unidos en los albores del siglo XXI, Zacatecas, México, Editorial Miguel Ángel Porrúa et université de Zacatecas, 2003.

14. ONU, World Population Prospects, op. cit.

15. Jorge Alonso et al., Lucha Urbana y Acumulación de Capital, México, éditions La Casa Chata, 1980.

16. Pablo Yankelevich, Deseables o inconvenientes. Las fronteras de la extranjería en el México posrevolucionario, Madrid, México, Bonilla Artigas Éditeurs, 2011.

17. Arias, Del arraigo a la diáspora. Dilemas de la familia rural, México, Editorial Miguel Angel Porrúa, 2009.

18. Jorge Durand et Douglas S. Massey, Clandestinos. Migración México Estados Unidos en los albores del siglo XXI, op. cit.

19. Manuel García y Griego et Mónica Verea, México y Estados Unidos frente a la Migración de Indocumentados, México, Miguel Angel Porrúa, 1998 ; Jorge Durand "Políticas emigratorias en un contexto de asimetría de poder. El caso mexicano, 1900-2003”, in Alberto Aziz Nassif y Jorge Alonso Sánchez (dir.), Globalización, poderes y seguridad nacional, México, H. Chambre des députés, LIX Legislatura-CIESAS-Miguel Angel Porrúa, 2005, pp. 105-131.

20. Douglas Massey, Jorge Durand et Nolan J. Malone, Detrás de la trama. Políticas migratorias entre México y Estados Unidos, México, Chambre des députés, LX Legislature-Université de Zacatecas, Miguel Ángel Porrúa, 2009. 